\title{
Generating Time Optimal Trajectory from Predefined 4D waypoint Networks
}

\author{
Kawser Ahmed, Kouamana Bousson
}

\begin{abstract}
The main purpose of this paper is to develop a trajectory optimization method to generate optimal trajectories that minimize aircraft total trip time between the initial and final waypoint in predefined 4D waypoint networks. In this paper, the 4D waypoint networks only consist of waypoints for climb, cruise and descent approach without the take-off and landing approach phases. The time optimal trajectory is generated for three different lengths of flights (short, medium, and long-haul flight) for two different commercial aircraft and considering zero wind condition. The Results about the presented applications show that by flying a time optimal trajectory, which was found by applying a single source shortest path algorithm (Dijkstra's algorithm), can lead to the reduction of average travel time by $2.6 \%$ with respect to the total trip time. Copyright () 2017 Praise Worthy Prize S.r.l. - All rights reserved.
\end{abstract}

Keywords: Time Conservation, Cost Index, 4D Waypoint Navigation, Trajectory Optimization, Dijkstra's Algorithm, Base of Aircraft Data (BADA)

\section{Nomenclature}

Acceleration

Earth semi major axis

Lift coefficient

Eccentricity

Waypoint

Earth radius

Wing area

Flight velocity

True air speed

Aircraft Nominal weight

Geocentric Coordinates

Flight path angle

Air density

Arrival time at waypoint

Heading
Travel time between waypoints

Graph, weight, and source vertex

Vertices and edge of the graph

Longitude, Latitude, and altitude

Arrival time tolerance interval

Distance between waypoints

\section{Introduction}

The air traffic load on the European air traffic network grows year upon year, the volume of air traffic is expected to double over the next 20 years.

Hence developing optimal trajectory that minimizes travel time on flight mission for commercial aircraft becomes an important factor nowadays not only because it helps the airlines to reduce their time related operating costs but also as it enhances air traffic flow in this rapidly growing aviation industry.

A practical solution that reduces the cost associated with time and fuel consumption during flight is the Cost Index $(\mathrm{CI})$. The value of the $\mathrm{CI}$ reflects the relative effects of fuel cost on overall trip cost as compared to time-related direct operating cost. For all the aircraft models, the minimum value of cost index equal to zero results in maximum range airspeed and minimum trip fuel, but this configuration ignores the time cost. If the cost index is maximum, the flight time is minimum, the velocity and the Mach number are maximum, but ignores the fuel cost [1]. In this paper, the Cost Index assumes to be maximum as only time cost is taken into consideration. The cost index is shown in equation (1):

$$
C I=\frac{\text { TimeCost } \sim(€ / \text { hour })}{\text { FuelCost } \sim(€ / \mathrm{kg})}
$$

Trajectory optimization is a vital area in aeronautic industry. This technique enables generating optimal trajectories for vehicles with consideration of fuel consumption, travel time and many other requirements. The trajectory optimization problem can be solved by optimal control methods. The optimal control problem can be solved by various kind of methods, however, these methods can be separated into two basic approaches: the indirect approach and the direct approach [2], [3].

The optimal control is solved by the Pontryagin maximum principle [4] in indirect approach, where the original optimal control problem is converted into EulerLagrange system (boundary value problem) by formulating the first order necessary condition which 
derived from Pontryagin maximum principle. Generally, the indirect approach leads to more accurate results than the direct approach. However, in indirect approach a good initial approximation of the co-state equation is needed in order to convergence, the physical meaning of co-state equation is not well established which make it difficult to guess [5]. Solving the optimal control problem by indirect approach also leads to two-point boundary value problem (TPBVPs), it demands computationally intensive iterative numerical procedures.

The direct approach is based on the transformation of optimal control problem into parameter optimization problem [6]. Which is done by discretizing the infinite dimensional problem into a finite dimensional problem and later on solving it by the nonlinear programming. Direct methods tend to have better convergence properties over indirect methods. Another great advantage of direct methods is that they do not have to deal with co-state equation. The most known direct approaches are based on Runge-Kutta scheme [7] and collocation methods [8]. Recently, some works have been presented for higher nonlinear dynamic system called a Chebyshev pseudo-spectral method [9], [10], [11].

Some research activities have been done for optimal trajectory generation in pre-defined network. Recently Boukraa, Bestaoui and Azouz [12] propose 3D optimal trim trajectories planner algorithm to generate trajectories for a set of predefined waypoints in space. However, the arrival time of each of these waypoints is not specified in their trajectory model. Bousson and Gameiro [13] present a quintic spline approach for 4D trajectory generation for UAVs. Devika and Thomas [14] present a path planning algorithm based on linear programming is adopted to ensure conflict free path in minimum time.

In this present paper, we suggest a new approach to generate 4D optimal trajectory defined by waypoints. In 4D trajectory the waypoints are consist of tri-dimensional coordinates as well as the arrival time in each waypoint. In this approach, the optimal trajectory is generated by applying greedy shortest path algorithms in graph theory, where the 4D waypoint networks are already prespecified. The shortest path algorithm approximates an optimal trajectory by the path that minimizes the total link cost connecting the origin and destination in a predefined network. This approach often require large computation time and memory space as the network grows bigger but the global optimal solutions is guaranteed to be found. In this paper, the single source shortest path algorithm (Dijkstra's algorithm) is used to generate the time optimal trajectory.

This study is restricted to the climb, cruise and descent phases of the flight and ignores the take-off and landing approach, and assuming the initial and final waypoints are at altitude of 3000 feet, where in the initial waypoint the aircraft begins the climb phase and in the final waypoint the aircraft begins the landing approach.

This paper primarily attempts to quantify benefits of time optimal trajectory which was generated by implying the Dijkstra's shortest path algorithm, here a benefit is meant to imply a reduction in total travel time due to using the Dijkstra's shortest path algorithm to the actual unimproved flight. The key aspect of this paper is a detailed comparison between actual flight trajectories and corresponding more efficient trajectory, thus giving the most realistic estimate of improvement potential.

\section{Problem Statement}

The main goal of this paper is to find a time optimal path from predefined 4D waypoint networks. A representation of waypoint networks is shown in Fig. 1, where $P_{1}$ is the initial waypoint and $P_{N}$ is the final waypoint of the networks.

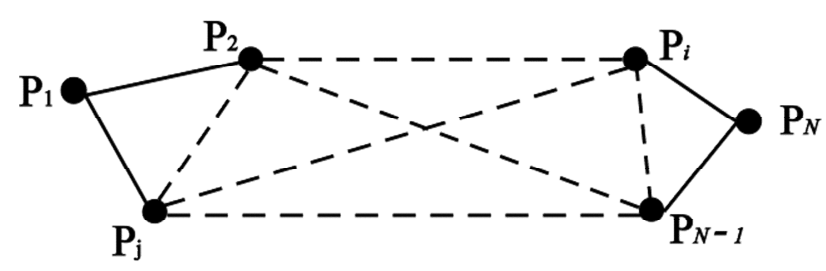

Fig. 1. Representation of 4D waypoint networks

Most of the approaches consider the waypoints defined by tri-dimensional coordinates positions as: $P_{k}=\left(\lambda_{k}, \varphi_{k}, h_{k}\right)^{T}$, where $k=1,2, \ldots, i, j, \ldots, N$ and do not take into account the arrival time $\tau_{k}$ at that waypoint. By adding the arrival time restriction, it is possible to define the 4D waypoints as: $P_{k}=\left(\lambda_{k}, \varphi_{k}, h_{k}, \tau_{k}\right)^{T}$. Where: $\lambda_{k}, \varphi_{k}, h_{k}, \tau_{k}$ is respectively longitude, latitude, altitude, and arrival time at waypoint $P_{k}$.

The problem to be solved is to estimate associated travel time $d \tau_{k}$ between the waypoints in 4D waypoint networks, then to use the value of associated travel time $d \tau_{k}$ between pair of waypoints as edges $\mathrm{E}$ and the waypoints as vertices $\mathrm{V}$ in Dijkstra's single source shortest path algorithm to generate the time optimal trajectory between the initial and final waypoints from the networks.

The following section propose a method that will generate the time optimal path along specified waypoints from predefined 4D waypoint networks by implying the Dijkstra's single source shortest path algorithm.

\section{Proposed Method}

To generate an optimal trajectory which minimizes travel time between the initial and final waypoints from a set of waypoints in 4D waypoint networks requires finding the associated travel time $d \tau_{k}$ by the aircraft to go from one waypoint to the other, defined as:

$$
d \tau_{k}=\tau_{k}-\tau_{k-1}
$$


where, $d \tau_{k}$ [min] is the travel time needed to go from waypoints $P_{k-1}$ to $P_{k}, \tau_{k}$ [min] is the travel time required to get to waypoint $P_{k}$ from initial waypoint and $\tau_{k-1}$ [min] is the travel time required to get to waypoint $P_{k-1}$ from initial waypoint.

In practice, the aircraft may not pass through the waypoint $P_{k}$ exactly at the specified time $\tau_{k}$ due to disturbances that may give rise to navigation inaccuracies. Therefore, an appropriate way is rather imposing a time tolerance interval $\varepsilon$ at each waypoint:

$$
\tau_{k}=\tau_{k-1}+\varepsilon \times d \tau_{k}
$$

where, $\varepsilon$ is the time tolerance interval for arrival at a determined waypoint $[1 \leq \varepsilon \leq 1.4]$. In the case when both waypoints $P_{k-1}$ and $P_{k}$ are at same altitude, the time tolerance interval $\varepsilon$ can be assumed as 1 .

\section{III.1. Dijkstra's Algorithm}

Dijkstra's algorithm, was first proposed by Dutch computer scientist Edsger Dijkstra in 1956 and published in 1959, is the most well-known shortest path algorithm. This is a graph search algorithm that solves the singlesource shortest path problem for a graph with nonnegative edge path costs, producing a shortest path tree. The most common variant of the algorithm fixes one vertex as the source and another as the destination vertex and find the shortest path between them.

Dijkstra's algorithm solves the single-source shortestpaths problem on a weighted, directed graph $\mathrm{G}(\mathrm{V}, \mathrm{E})$ where $\mathrm{V}$ is a set of vertices and $\mathrm{E}$ is a set of edges on the graph. This algorithm requires 3 variables as input in order to finds the path with lowest cost between the source and destination vertices, they are respectively the graph, the source vertex, and the destination vertex, and at the end it returns a reduced graph as output.

This algorithm will determine the global optimal (best route to take), given a number of vertices and edges as long as it has the graph as an input, no matter how large the graph is.

In addition to the basic formulation of the Dijkstra's algorithm, the following aspects must be defined specifically for the flight trajectory optimization problem. The number of vertices $\mathrm{V}$, the edges $\mathrm{E}$ between the vertices and the source and destination vertices. In this paper, the waypoints of the 4D waypoint networks are the vertices $\mathrm{V}$, the initial waypoint is the source vertex $\mathrm{s}$, the final waypoint is the destination vertex and the associated travel time $d \tau_{k}$ by the aircraft between the pairs of waypoints are the edges $E$ between these vertices (waypoints).

In Figs. 2 a full execution of the Dijkstra's shortest path algorithm operation is shown. The circles represent the vertices or nodes and the lines with arrows are the edges.
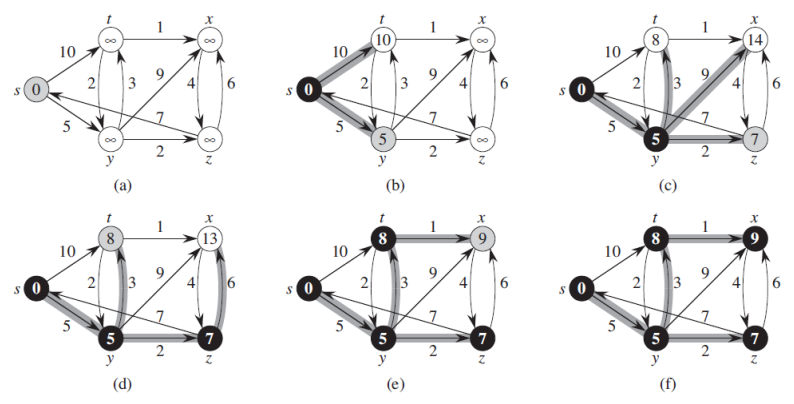

Figs. 2. The execution of Dijkstra's algorithm

Each edge has a non-negative cost associated with it. The problem is to find the most cost-efficient route from the source vertex to any other vertex.

In this example, the source vertex $s$ is the leftmost vertex. The value with low cost estimates appear within the vertices, and shaded edges indicate predecessor values. Black vertices are already examined thus they have the value of lowest cost associated with them to go from the source vertex, and the white vertices are going to be examined. First step (a) shows the situation just before the first iteration of the while loop. Form step (b) to step (f) shows the situation after each successive iteration of the while loop. The value of lowest cost and predecessors shown in last step (f), and these are the final values of the lowest cost to go to that vertex from the source vertex [15], [16], [17].

\section{III.2. Modelling of 4D Waypoint Network}

Assuming the 4D waypoint networks consists of $\mathrm{N}$ sets of waypoints, where $P_{1}$ is the initial waypoint and $P_{N}$ is the final waypoint in the waypoint networks. Each waypoint $P_{k}$, where $k=1,2, \ldots, i, j, \ldots, N$ is defined by the geodetic coordinates $\lambda_{k}, \varphi_{k}, h_{k}$, by considering the arrival time in each waypoint, the waypoint $P_{k}$ can be described as a four-dimensional state vector:

$$
P_{k}=\left(\lambda_{k}, \varphi_{k}, h_{k}, \tau_{k}\right)^{T}
$$

where, $\lambda_{k}$ is the longitude, $\varphi_{k}$ is the latitude, $h_{k}$ is the altitude (with respect to sea level) and $\tau_{k}$ is the scheduled arrival time at waypoint $P_{k}$. The following subsections represent the navigation model and constraints of 4D waypoint networks.

\section{III.2.1. Navigation Model}

The following differential equations model the dynamics of the navigation process:

$$
\dot{\lambda}=\frac{V \cos \gamma \sin \psi}{\left(R_{e}+h\right) \cos \varphi}
$$




$$
\begin{gathered}
\dot{\varphi}=\frac{V \cos \gamma \cos \psi}{\left(R_{e}+h\right)} \\
\dot{h}=V \sin \gamma \\
\dot{V}=u_{1} \\
\dot{\gamma}=u_{2} \\
\dot{\psi}=u_{3}
\end{gathered}
$$

where, $V$ is the flight velocity, $\gamma$ is the flight path angle, $\psi$ is the heading (with respect to the geographical north), $\lambda$ is the longitude, $\varphi$ is the latitude, $h$ is the altitude (with respect to sea level) and $R_{e}$ is the Earth radius.

The variables $u_{1}, u_{2}$ and $u_{3}$ are respectively the acceleration, the flight path angle rate, and the heading rate. The state vector $x$ and control vector $u$ of the above model are described respectively as:

$$
\begin{gathered}
x=(\lambda, \varphi, h, V, \gamma, \psi)^{T} \\
u=\left(u_{1}, u_{2}, u_{3}\right)^{T}
\end{gathered}
$$

\section{III.2.2. Navigation Constraints}

The real-world flight operates under several constraints, due to aircraft performance, aerodynamic, structural limits, safety reasons, the mission and other factor, which is described as:

$$
\begin{aligned}
& a_{\text {min }} \leq a \leq a_{\text {max }} \\
& \gamma_{\text {min }} \leq \gamma \leq \gamma_{\text {max }} \\
& V_{\text {min }} \leq V \leq V_{\text {max }}
\end{aligned}
$$

These bound constraints are imposed on the state and control vectors.

\section{III.3. Arrival Time at Each Waypoint}

The 4D navigation consists of traveling through a sequence of predefined waypoints in a given time of flight, which in turn define the trajectory of the flight. Assuming that the waypoint $P_{k}$ is already defined by the geodetic coordinates longitude $\lambda_{k}$, latitude $\varphi_{k}$, and altitude $h_{k}$, where the scheduled time of arrival $\tau_{k}$ at this waypoint is unknown. To compute this unknown arrival time of each waypoint, the distance between this waypoint and its previous waypoint from where the aircraft is arriving and the average velocity of the aircraft between these two waypoints are required.

The trajectory generation requires a geocentric coordinates system. To calculate the distance between two waypoints, the tri-dimensional waypoints need to be transformed from usual geodetic coordinates system to geocentric coordinates system. The $3 \mathrm{D}$ waypoint $P_{k}$ is defined by the following way:

$$
P_{k}=\left(\lambda_{k}, \varphi_{k}, h_{k}\right)^{T}
$$

Now to transform these geodetic coordinates to geocentric coordinates, the following equations are required [18]:

$$
\begin{gathered}
X_{k}=\left(N_{k}+h_{k}\right) \cos \varphi_{k} \cos \lambda_{k} \\
Y_{k}=\left(N_{k}+h_{k}\right) \cos \varphi_{k} \sin \lambda_{k} \\
Z_{k}=\left[N_{k}\left(1-e^{2}\right)+h_{k}\right] \sin \varphi_{k}
\end{gathered}
$$

Being $\mathrm{a}$ is the Earth semi major axis and $e$ its eccentricity, $N_{k}$ can be calculated as follows:

$$
N_{k}=\frac{\mathrm{a}}{\sqrt{1-e^{2} \sin ^{2} \varphi_{k}}}
$$

After transforming the 3D waypoints from geodetic coordinates to geocentric coordinates system, now it is possible to calculate $\Delta d_{k}$ (the distance between two waypoints $P_{k-1}$ and $P_{k}$ ) by using the following equation:

$$
\Delta d_{k}=\sqrt{\left(X_{k}-X_{k-1}\right)^{2}+\left(Y_{k}-Y_{k-1}\right)^{2}+\left(Z_{k}-Z_{k-1}\right)^{2}}
$$

To estimate the appropriate velocity of the aircraft $V_{k}$ at any waypoint $P_{k}$, the following equation can be used:

$$
V_{k}=\sqrt{\frac{2 W}{\rho_{k} C_{L} S}}
$$

where, $W$ is the aircraft nominal weight, $C_{L}$ is the lift coefficient, $S$ is the wing area of the aircraft and $\rho_{k}$ is the air density (varies with altitude) at waypoint $P_{k}$. It is possible to get the appropriate velocity at any waypoint $P_{k}$ from Base of Aircraft Data (BADA), where true air speed, VTAS [kt] is specified for different aircraft for different flight level and phases of the flights [19].

By using the distance between two waypoints and the velocity of the aircraft in both waypoints the travel time between these two waypoints can be computed as follow: 


$$
d \tau_{k}=\frac{\Delta d_{k}}{\frac{V_{k}+V_{k-1}}{2}}
$$

where, $d \tau_{k}$ is the time needed to go from waypoint $P_{k-1}$ to $P_{k}$.

\section{Simulation and Result}

In this section, the simulation and result of the time optimal trajectory is shown for three different length (short, medium, and long-haul) of flight considering zero wind conditions. All the analysis of the simulation has been done using Matlab 2013 ${ }^{\mathrm{a}}$. The coordinates of the trajectory were chosen using sky vector website [20].

\section{IV.1. Short-Haul Flight}

In order to analyze the short-haul flight, the flight from Lisbon to Geneva was considered. The 4D waypoint networks of this short-haul flight consists of two different trajectories, and has total 22 waypoints including the initial and final waypoints, and each trajectory has 12 waypoints including the initial and final waypoints. Airplane A1 (which is a short to medium range narrow body twinjet airliner) was used to analyze the flight trajectories. Tables I and II show the waypoints lists for both of the trajectories. Each waypoint is defined in geodetic coordinates $(\lambda, \varphi, h)$, and their associated distance travel time $d \tau_{k}$ between the waypoints are also shown.

The trajectories were chosen such a way that the climb and descent phases of the first trajectory are smaller than the second trajectory but the cruise phase of the first trajectory is bigger than the second trajectory, therefore the total distance from the initial to final waypoint for both of the trajectories are more or less same. The time optimal trajectory was generated from the 4D waypoint networks by using the Dijkstra's single source shortest path algorithm.

TABLE I

LIST OF WAYPOINTS IN 1ST TRAJECTORY FOR SHORT-HAUL FLIGHT

\begin{tabular}{ccccc}
\hline \hline waypoint & $\begin{array}{c}\lambda \\
{[\mathrm{deg}]}\end{array}$ & $\begin{array}{c}\varphi \\
{[\mathrm{deg}]}\end{array}$ & $\begin{array}{c}h \\
{[\mathrm{feet}]}\end{array}$ & $\begin{array}{c}d \tau_{k} \\
{[\mathrm{~min}]}\end{array}$ \\
\hline Initial $\left(\mathrm{P}_{1}\right)$ & -9.0405 & 38.9955 & 3000 & 0 \\
$\mathrm{P}_{2}$ & -8.9083 & 39.1087 & 10000 & 2.371751 \\
$\mathrm{P}_{3}$ & -8.624 & 39.33417 & 20000 & 3.437191 \\
$\mathrm{P}_{4}$ & -7.7987 & 39.8783 & 33000 & 7.295251 \\
$\mathrm{P}_{5}$ & -6.9993 & 40.2513 & 39000 & 5.748997 \\
$\mathrm{P}_{6}$ & -3.3707 & 43.227 & 39000 & 32.48854 \\
$\mathrm{P}_{7}$ & 0.1303 & 44.729 & 39000 & 23.71969 \\
\hline & $\lambda$ & $\varphi$ & $h$ & $d \tau_{k}$ \\
waypoint & {$[\mathrm{deg}]$} & {$[\mathrm{deg}]$} & {$[\mathrm{feet}]$} & {$[\mathrm{min}]$} \\
\hline $\mathrm{P}_{8}$ & 3.5963 & 44.9543 & 39000 & 19.97808 \\
$\mathrm{P}_{9}$ & 3.87217 & 45.1205 & 33000 & 2.058735 \\
$\mathrm{P}_{10}$ & 4.6985 & 45.41983 & 20000 & 5.71202 \\
$\mathrm{P}_{11}$ & 5.336 & 45.606 & 10000 & 5.275779 \\
Final $\left(\mathrm{P}_{22}\right)$ & 5.7553 & 45.884 & 3000 & 5.618537 \\
\hline Total & \multicolumn{5}{c}{} & 113.7046 \\
\hline \hline
\end{tabular}

TABLE II

LIST OF WAYPOINTS IN 2ND TRAJECTORY FOR SHORT-HAUL FLIGHT

\begin{tabular}{ccccc}
\hline \hline waypoint & $\begin{array}{c}\lambda \\
{[\mathrm{deg}]}\end{array}$ & $\begin{array}{c}\varphi \\
{[\mathrm{deg}]}\end{array}$ & $\begin{array}{c}h \\
{[\mathrm{feet}]}\end{array}$ & $\begin{array}{c}d \tau_{k} \\
{[\mathrm{~min}]}\end{array}$ \\
\hline Initial $\left(\mathrm{P}_{1}\right)$ & -9.0405 & 38.9955 & 3000 & 0 \\
$\mathrm{P}_{12}$ & -8.835 & 39.0883 & 10000 & 2.862798 \\
$\mathrm{P}_{13}$ & -8.49983 & 39.30183 & 20000 & 3.66612 \\
$\mathrm{P}_{14}$ & -7.59217 & 39.7763 & 33000 & 7.374258 \\
$\mathrm{P}_{15}$ & -6.715 & 40.11317 & 39000 & 6.036433 \\
$\mathrm{P}_{16}$ & -1.765 & 41.631 & 39000 & 32.6647 \\
$\mathrm{P}_{17}$ & 0.5565 & 43.9993 & 39000 & 23.55432 \\
$\mathrm{P}_{18}$ & 3.6277 & 44.85317 & 39000 & 19.04446 \\
$\mathrm{P}_{19}$ & 3.9993 & 44.9983 & 33000 & 2.415762 \\
$\mathrm{P}_{20}$ & 4.8617 & 45.261 & 20000 & 5.787862 \\
$\mathrm{P}_{21}$ & 5.4975 & 45.5203 & 10000 & 5.625951 \\
Final $\left(\mathrm{P}_{22}\right)$ & 5.7553 & 45.884 & 3000 & 5.642634 \\
\hline Total
\end{tabular}

The time optimal trajectory contains 10 waypoints [initial waypoint $\left(\mathrm{P}_{1}\right) \rightarrow \mathrm{P}_{2} \rightarrow \mathrm{P}_{3} \rightarrow \mathrm{P}_{4} \rightarrow \mathrm{P}_{5} \rightarrow \mathrm{P}_{8} \rightarrow \mathrm{P}_{9} \rightarrow$ $\mathrm{P}_{10} \rightarrow \mathrm{P}_{11} \rightarrow$ final waypoint $\left.\left(\mathrm{P}_{22}\right)\right]$, the distance between the initial and final waypoints in the time optimal trajectory is $777.8 \mathrm{~nm}$. The comparison of travel time in different phases of flight for those two trajectories and time optimal trajectory is shown in (Table III).

TABLE III

TOTAL TIME NEEDED IN DIFFERENT TRAJECTORIES FOR SHORT-HAUL FLIGHT

\begin{tabular}{ccccc}
\hline \hline \multirow{2}{*}{ Trajectory } & \multicolumn{3}{c}{ Time [min] } & $\begin{array}{c}\text { Total } \\
{[\mathrm{min}]}\end{array}$ \\
\cline { 2 - 4 } & Climb & Cruise & Descent & 113.7 \\
1 & 18.9 & 76.2 & 18.7 & 114.7 \\
2 & 19.9 & 75.3 & 19.5 & 111.01 \\
\hline \hline
\end{tabular}

As it is seen from Table III that by using the time optimal trajectory in short-haul flight (Lisbon - Geneva), the aircraft reaches the final waypoint 2.7 minutes faster than the first trajectory, which is equivalent to $2.4 \%$ of total travel time of the first trajectory and 3.7 minutes faster than the second trajectory equivalent to $3.2 \%$ of total travel time of the second trajectory. The time optimal trajectory of short-haul flight is shown in Fig. 3.

In Fig. 3 the blue curve line represents the real trajectory path through different waypoints, starting on the right. The red circles around the blue line denote the position of the waypoints associated with the trajectory.

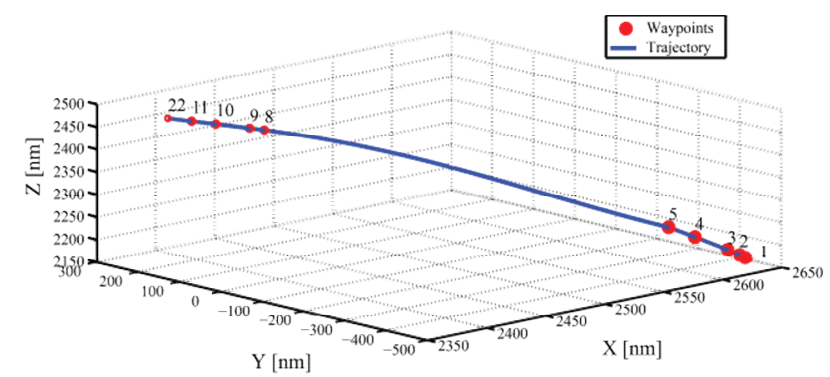

Fig. 3. 3D time optimal trajectory in geocentric coordinates for short-haul flight

\section{IV.2. Medium-Haul Flight}

To analyze the medium-haul flight, the flight from Lisbon to Stockholm was considered. 
There are also two trajectories between the initial and final waypoints in the 4D waypoint networks, each trajectory has total 13 waypoints including the initial and final waypoints, and total 24 waypoints are there in the 4D networks including the initial and final waypoint. Airplane A2 (which is a long range wide body twinjet airliner) was used to analyze the flight trajectories.

Tables IV and V show the waypoints lists for both trajectories.

TABLE IV

LIST OF WAYPOINTS IN 1ST TRAJECTORY

FOR THE MEDIUM-HAUL FLIGHT

\begin{tabular}{ccccc}
\multicolumn{5}{c}{ FOR THE MEDIUM-HAUL FLIGHT } \\
\hline \hline waypoint & $\begin{array}{c}\lambda \\
{[\mathrm{deg}]}\end{array}$ & $\begin{array}{c}\varphi \\
{[\mathrm{deg}]}\end{array}$ & $\begin{array}{c}h \\
{[\mathrm{feet}]}\end{array}$ & $\begin{array}{c}d \tau_{k} \\
{[\mathrm{~min}]}\end{array}$ \\
\hline Initial $\left(\mathrm{P}_{1}\right)$ & -9.0405 & 38.9955 & 3000 & 0 \\
$\mathrm{P}_{2}$ & -8.9373 & 39.1525 & 10000 & 2.665515 \\
$\mathrm{P}_{3}$ & -8.643 & 39.6385 & 24000 & 5.529098 \\
$\mathrm{P}_{4}$ & -8.0007 & 40.5407 & 37000 & 8.291856 \\
$\mathrm{P}_{5}$ & -7.7847 & 40.942 & 41000 & 3.243938 \\
$\mathrm{P}_{6}$ & -4.752 & 46.1707 & 41000 & 42.43539 \\
$\mathrm{P}_{7}$ & 1.07817 & 49.6497 & 41000 & 39.21055 \\
$\mathrm{P}_{8}$ & 9.07617 & 53.515 & 41000 & 47.18143 \\
$\mathrm{P}_{9}$ & 14.4575 & 57.839 & 41000 & 39.59531 \\
$\mathrm{P}_{10}$ & 14.75417 & 58.0005 & 37000 & 1.695762 \\
$\mathrm{P}_{11}$ & 15.78983 & 58.624 & 24000 & 6.807662 \\
$\mathrm{P}_{12}$ & 17.0113 & 59.2063 & 10000 & 9.043191 \\
Final $\left(\mathrm{P}_{24}\right)$ & 17.73983 & 59.3445 & 3000 & 5.538229 \\
\hline Total & \multicolumn{5}{c}{} \\
\hline \hline
\end{tabular}

TABLE V

LIST OF WAYPOINTS IN 2ND TRAJECTORY FOR THE MEDIUM-HAUL FLIGHT

\begin{tabular}{|c|c|c|c|c|}
\hline waypoint & $\begin{array}{c}\lambda \\
{[\mathrm{deg}]}\end{array}$ & $\begin{array}{c}\varphi \\
{[\mathrm{deg}]}\end{array}$ & $\begin{array}{c}h \\
{[\text { feet] }}\end{array}$ & $\begin{array}{c}d \tau_{k} \\
{[\mathrm{~min}]}\end{array}$ \\
\hline Initial $\left(\mathrm{P}_{1}\right)$ & -9.0405 & 38.9955 & 3000 & 0 \\
\hline $\mathrm{P}_{13}$ & -8.8405 & 39.1047 & 10000 & 2.874146 \\
\hline $\mathrm{P}_{14}$ & -8.455 & 39.576 & 24000 & 5.748028 \\
\hline $\mathrm{P}_{15}$ & -7.808 & 40.4545 & 37000 & 8.141368 \\
\hline $\mathrm{P}_{16}$ & -7.413 & 40.8377 & 41000 & 3.642769 \\
\hline $\mathrm{P}_{17}$ & -0.2773 & 44.76 & 41000 & 49.00066 \\
\hline waypoint & $\begin{array}{c}\lambda \\
{[\mathrm{deg}]}\end{array}$ & $\begin{array}{c}\varphi \\
{[\mathrm{deg}]}\end{array}$ & $\begin{array}{c}h \\
{[\text { feet] }}\end{array}$ & $\begin{array}{c}d \tau_{k} \\
{[\min ]}\end{array}$ \\
\hline $\mathrm{P}_{18}$ & 4.6187 & 50.023 & 41000 & 46.56667 \\
\hline $\mathrm{P}_{19}$ & 10.92483 & 54.85883 & 41000 & 46.27695 \\
\hline $\mathrm{P}_{20}$ & 15.0405 & 57.2845 & 41000 & 25.06379 \\
\hline $\mathrm{P}_{21}$ & 15.39 & 57.4757 & 37000 & 2.016462 \\
\hline $\mathrm{P}_{22}$ & 16.496 & 58.17 & 24000 & 7.488843 \\
\hline $\mathrm{P}_{23}$ & 17.504 & 58.94117 & 10000 & 9.827318 \\
\hline Final $\left(\mathrm{P}_{24}\right)$ & 17.73983 & 59.3445 & 3000 & 5.864875 \\
\hline Total & & & & 212.5119 \\
\hline
\end{tabular}

The time optimal trajectory for the flight between Lisbon and Stockholm (medium-haul flight) contains 9 waypoints [initial waypoint $\left(\mathrm{P}_{1}\right) \rightarrow \mathrm{P}_{2} \rightarrow \mathrm{P}_{3} \rightarrow \mathrm{P}_{4} \rightarrow \mathrm{P}_{5} \rightarrow$ $\mathrm{P}_{9} \rightarrow \mathrm{P}_{10} \rightarrow \mathrm{P}_{12} \rightarrow$ final waypoint $\left.\left(\mathrm{P}_{24}\right)\right]$, the distance between the initial and final waypoints in time optimal trajectory is $1589.6 \mathrm{~nm}$.

The comparison of travel time in different phases of the flight for those two trajectories and the time optimal trajectory is shown in Table VI.

It can be seen from Table VI that by flying the time optimal trajectory in medium-haul flight (Lisbon Stockholm) 4.2 minutes can be saved than the first trajectory, which is equivalent to $1.9 \%$ of total travel time of the first trajectory and 5.5 minutes can be saved than the second trajectory, which is equivalent to $2.6 \%$ of total travel time of the second trajectory. The time optimal trajectory is shown in Fig. 4.

In Fig. 4 the blue curved line is the time optimal trajectory path and the red circles around it are the associated waypoints of the time optimal trajectory.

TABLE VI

TOTAL TIME NEEDED IN DIFFERENT TRAJECTORIES FOR MEDIUM-HAUL FLIGHT

\begin{tabular}{ccccc}
\hline \hline \multirow{2}{*}{ Trajectory } & \multicolumn{3}{c}{ Time [min] } & $\begin{array}{c}\text { Total } \\
{[\mathrm{min}]}\end{array}$ \\
\cline { 2 - 4 } & Climb & Cruise & Descent & 211.2 \\
2 & 19.7 & 168.4 & 23.1 & 212.5 \\
Time optimal & 20.4 & 166.9 & 25.2 & 207.04 \\
\hline \hline
\end{tabular}

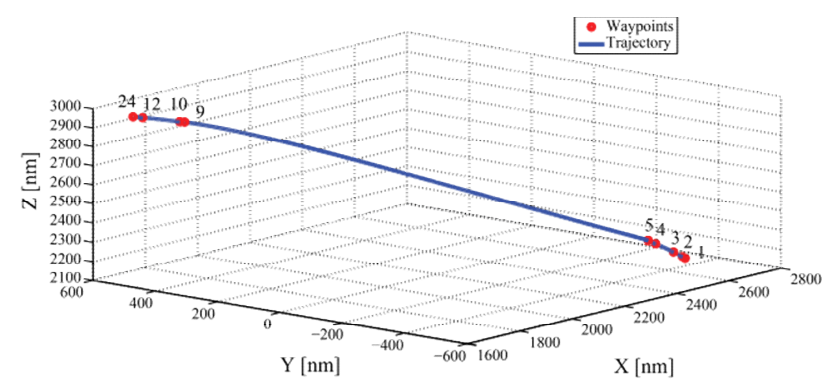

Fig. 4. 3D time optimal trajectory in geocentric coordinates for medium-haul flight

\section{IV.3. Long-Haul Flight}

The flight from Lisbon to Montreal was considered in order to analyze the long-haul flight.

The 4D waypoint networks of the long-haul flight also consists of two trajectories between the initial and final waypoints, each trajectory has 14 waypoints including the initial and final waypoints, and total 26 waypoints are in the whole 4D waypoint networks including initial and final waypoints.

Airplane A2 (which is a long range wide body twinjet airliner) was used to analyze the flight trajectories. The waypoints list of both trajectories is shown in Table VII and VIII.

TABLE VII

LIST OF WAYPOINTS IN 1ST TRAJECTORY FOR THE LONG-HAUL FLIGHT

\begin{tabular}{ccccc}
\hline \hline waypoint & $\begin{array}{c}\lambda \\
{[\mathrm{deg}]}\end{array}$ & $\begin{array}{c}\varphi \\
{[\mathrm{deg}]}\end{array}$ & $\begin{array}{c}h \\
{[\mathrm{feet}]}\end{array}$ & $\begin{array}{c}d \tau_{k} \\
{[\mathrm{~min}]}\end{array}$ \\
\hline Initial $\left(\mathrm{P}_{1}\right)$ & -9.0405 & 38.9955 & 3000 & 0 \\
$\mathrm{P}_{2}$ & -9.22083 & 39.1 & 10000 & 2.64759 \\
$\mathrm{P}_{3}$ & -9.8425 & 39.3857 & 24000 & 5.780186 \\
$\mathrm{P}_{4}$ & -10.9352 & 39.9543 & 37000 & 8.209056 \\
$\mathrm{P}_{5}$ & -11.74 & 40.32817 & 43000 & 5.40015 \\
$\mathrm{P}_{6}$ & -22.5022 & 43.5023 & 43000 & 64.53132 \\
$\mathrm{P}_{7}$ & -35.132 & 44.73617 & 43000 & 68.56969 \\
$\mathrm{P}_{8}$ & -44.9847 & 47.4295 & 43000 & 55.05948 \\
$\mathrm{P}_{9}$ & -58.667 & 48.5647 & 43000 & 69.12781 \\
$\mathrm{P}_{10}$ & -70.3565 & 46.4947 & 43000 & 61.15854 \\
$\mathrm{P}_{11}$ & -70.809 & 46.4135 & 37000 & 2.421801 \\
$\mathrm{P}_{12}$ & -71.9328 & 46.21 & 24000 & 6.6038 \\
$\mathrm{P}_{13}$ & -73.0908 & 45.9335 & 10000 & 8.948898 \\
Final $\left(\mathrm{P}_{26}\right)$ & -73.6412 & 45.7947 & 3000 & 5.680405 \\
\hline Total & \multicolumn{5}{c}{} & 364.1387 \\
\hline \hline
\end{tabular}


TABLE VIII

LIST OF WAYPOINTS IN 2ND TRAJECTORY FOR THE LONG-HAUL FLIGHT

\begin{tabular}{|c|c|c|c|c|}
\hline waypoint & $\begin{array}{c}\lambda \\
{[\mathrm{deg}]}\end{array}$ & $\begin{array}{c}\varphi \\
{[\mathrm{deg}]}\end{array}$ & $\begin{array}{c}h \\
\text { [feet] }\end{array}$ & $\begin{array}{c}d \tau_{k} \\
{[\mathrm{~min}]}\end{array}$ \\
\hline Initial $\left(\mathrm{P}_{1}\right)$ & -9.0405 & 38.9955 & 3000 & 0 \\
\hline $\mathrm{P}_{14}$ & -9.2387 & 39.05683 & 10000 & 2.514717 \\
\hline $\mathrm{P}_{15}$ & -9.94583 & 39.2603 & 24000 & 6.040968 \\
\hline $\mathrm{P}_{16}$ & -11.2588 & 39.4515 & 37000 & 8.362034 \\
\hline $\mathrm{P}_{17}$ & -11.9673 & 39.9493 & 43000 & 5.533107 \\
\hline $\mathrm{P}_{18}$ & -24.8818 & 42.095 & 43000 & 74.7246 \\
\hline $\mathrm{P}_{19}$ & -34.374 & 43.996 & 43000 & 53.92886 \\
\hline $\mathrm{P}_{20}$ & -44.3497 & 46.44083 & 43000 & 55.77103 \\
\hline $\mathrm{P}_{21}$ & -61.77 & 47.44 & 43000 & 89.28184 \\
\hline $\mathrm{P}_{22}$ & -70.163 & 46.2543 & 43000 & 43.99628 \\
\hline $\mathrm{P}_{23}$ & -70.557 & 46.102 & 37000 & 2.317716 \\
\hline $\mathrm{P}_{24}$ & -71.739 & 45.9043 & 24000 & 6.98395 \\
\hline $\mathrm{P}_{25}$ & -72.9952 & 45.81 & 10000 & 9.270174 \\
\hline \multirow{2}{*}{ waypoint } & $\lambda$ & $\varphi$ & $h$ & $d \tau_{k}$ \\
\hline & [deg] & [deg] & [feet] & [min] \\
\hline Final $\left(\mathrm{P}_{26}\right)$ & -73.6412 & 45.7947 & 3000 & 6.280807 \\
\hline Total & & & & 365.0061 \\
\hline
\end{tabular}

The time optimal trajectory of flight between Lisbon to Montreal (long-haul flight) contains 9 waypoints [initial waypoint $\left(\mathrm{P}_{1}\right) \rightarrow \mathrm{P}_{14} \rightarrow \mathrm{P}_{3} \rightarrow \mathrm{P}_{4} \rightarrow \mathrm{P}_{5} \rightarrow \mathrm{P}_{10} \rightarrow$ $\mathrm{P}_{11} \rightarrow \mathrm{P}_{13} \rightarrow$ final waypoint $\left(\mathrm{P}_{26}\right)$, the distance between the initial and final waypoints in time optimal trajectory of this flight is $2772 \mathrm{~nm}$. The comparison of travel time in different phases of flight for different trajectories and time optimal trajectory is shown in Table IX.

Table IX suggest that by using the time optimal trajectory in long-haul flight (Lisbon - Montreal) the aircraft reaches to the final waypoint from the initial waypoint 9.9 minutes faster than the first trajectory which saves $2.7 \%$ of the total travel time and 10.8 minutes faster than the second trajectory which save $2.9 \%$ of total travel time. The time optimal trajectory of long-haul flight in 3D is shown in Fig. 5.

TABLE IX

TOTAL TIME NEEDED IN DIFFERENT TRAJECTORIES FOR LONG-HAUL FLIGHT

\begin{tabular}{ccccc}
\hline \hline Trajectory & \multicolumn{3}{c}{ Time [min] } & $\begin{array}{c}\text { Total } \\
{[\mathrm{min}]}\end{array}$ \\
\cline { 2 - 4 } & Climb & Cruise & Descent & 364.2 \\
1 & 22.04 & 318.5 & 23.7 & 365.1 \\
2 & 22.5 & 317.7 & 24.9 & 354.3 \\
\hline \hline
\end{tabular}

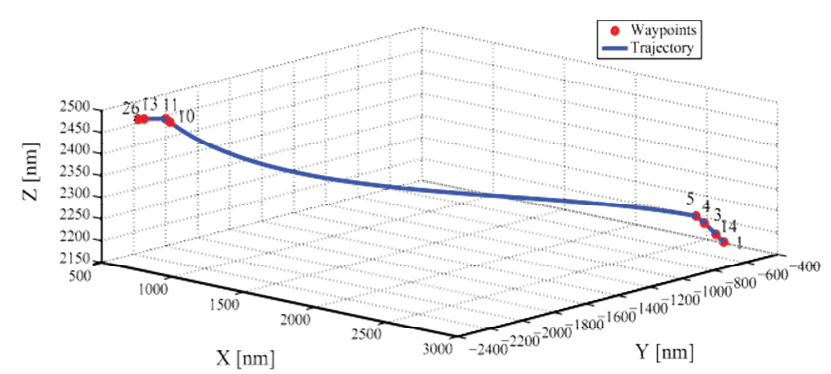

Fig. 5. 3D time optimal trajectory in geocentric coordinates for long-haul flight

In Fig. 5 the blue curve is the time optimal trajectory and the red circles around it are the waypoints of the trajectory. In this long-haul flight, the cruise phase is large compare to its climb and descent phases, thus in the fig. of time optimal trajectory for this flight the waypoints in the climb and descent phases are seems too close to each other.

\section{Conclusion}

This paper is based on finding the time optimal trajectory of climb, cruise, and descent phases of the flight, but ignores the take-off and landing phases of the flight. In this study, several steps were made in order to achieve a complete trajectory from predefined 4D waypoint networks that optimize the total travel time.

This study uses Dijkstra's single source shortest path algorithm in order to find the time optimal trajectory. Later on, this time optimal trajectory was used to compare with the existing 2 trajectories of different length (short, medium and long-haul) of flights.

The analysis results show promising potential for reduction of travel time in different flights via using the Dijkstra's single source shortest path algorithm, across a range of common aircrafts and routes. The results suggest that by flying time optimal trajectory for shorthaul flight, it is possible to save $2.7-3.7$ minutes of travel time which is equivalent to $2.4-3.2 \%$ of total travel time. In medium-haul flight by flying the time optimal trajectory the travel time was reduced by $4.2-$ 5.5 minutes or $1.9-2.6 \%$ of total travel time. For longhaul flight, it is possible to save $9.9-10.8$ minutes or 2.7 $-2.9 \%$ of total travel time by flying the time optimal trajectory. In general, the savings of the travel time are proportional to the trip lengths, and depends on the aircraft types. Despite of the fact that the algorithm has proven reliable to find the time optimal trajectory from pre-defined 4D waypoint networks, there is still room for improvement. By using more trajectories with different cruise altitude in the networks more travel time can be saved, as there will be more waypoints to choose from. However, this approach is not well suited for online trajectory optimization as the waypoint networks need to be pre-defined which requires some amount of time. But this can be solved by generating the waypoint networks by direct optimal control methods.

In addition, a realistic wind model and Air traffic control (ATC) restrictions can be imposed on the 4D waypoint networks to model more realistic flight.

\section{Acknowledgements}

This research work was conducted in the Aeronautics and Astronautics Research Group (AeroG) of the Associated Laboratory for Energy, Transports and Aeronautics (LAETA).

\section{References}

[1] Roberson B, "Fuel Conservation Strategies: Cost Index Explained," Boeing, 2007, pp. 26-28. 
[2] Bryson A. E and Ho Y. C, "Applied Optimal Control: Optimization, Estimation and, Control", Taylor \& Francis, New York, 1975

[3] Betts, J.T., "Survey of numerical methods for trajectory optimization" Journal of Guidance, Control and Dynamics, 1998, pp. 193-207.

[4] Pontryagin, L.S., V.G. Boltyanskii, R.V. Gamkrelidze, E.F. Mishchenko. The Mathematical Theory of Optimal Processes, Wiley-Interscience, New-York, 1962.

[5] Von Stryk, O. and R. Bulirsch. "Direct and indirect methods for trajectory optimization", Annals of Operations Research, 37, 1992, pp. 357-373.

[6] Hull, D.G., "Conversion of Optimal Control Problems into Parameter Optimization Problems", Journal of Guidance, Control, and Dynamics, 1997, pp. 57-60.

[7] Schwartz, A. and E. Polak, "Consistent approximations for Optimal Control Problems Based on Runge-Kutta Integration", SIAM Journal on Control and optimization, vol.34, No.4, 1996, pp. $1235-1269$.

[8] Hargraves, C.R. and S.W. Paris, "Direct Trajectory Optimization Using Nonlinear Programming and Collocation", Journal of Guidance, Control and Dynamics, vol. 10, No.4, 1987, pp. 338342

[9] Bousson, K. "Chebyshev pseudospectral trajectory optimization of differential inclusion models", SAE World Aviation Congress, Montreal, Canada, paper no. 2003-01-3044, 2003.

[10] Fahroo, F. and I.M. Ross, "Direct trajectory optimization by a Chebyshev pseudospectral method", Journal of Guidance, Control and Dynamics, 2002, pp. 160-166.

[11] Bousson K. and Machado P, "4D Flight Trajectory Generation and Tracking for Waypoint-Based Aerial Navigation," WSEAS transactions on system and control, Vol 8, No 3, july 2013, pp 105-119.

[12] Boukraa, D., Bestaoui, Y., Azouz, N., Three Dimensional Trajectory Generation for an Autonomous Plane, (2014) International Journal on Numerical and Analytical Methods in Engineering (IRENA), 2 (4), pp. 144-154.

[13] Bousson, K., Gameiro, T., A Quintic Spline Approach to 4D Trajectory Generation for Unmanned Aerial Vehicles, (2015) International Review of Aerospace Engineering (IREASE), 8 (1), pp. 1-9.

[14] Devika, K., Thomas, S., Path Planning for Conflict Resolution in Free Flight System: Optimization Based on Linear Programming, (2016) International Review of Aerospace Engineering (IREASE), 9 (1), pp. 1-6.

[15] Cormen T. H, Leiserson C. E, Rivest R. L and Stein C, "Introduction to algorithms", London, England: The MIT press, 2009, pp. 658-659.

[16] Hart C, "Graph Theory Topics in Computer Networking," (2013, pp 13-20).

[17] Dasgupta S, Papadimitriou C. H and Vazirani U. V, "Algorithms", McGraw-Hill, New York, July 18, 2006, pp. 112-118.

[18] Seemkooei A. A, "Comparison of different algorithm to transform geocentric to geodetic coordinates," Survey Review 36, October 2002, pp 627-632.

[19] EUROCONTROL "Aircraft Performance Summary Tables for the Base of Aircraft Data (BADA)," Eurocontrol Experimental Centre, Revision 3.4, June 2002.

[20] "SkyVector," Website Available: https://skyvector.com/. [Accessed October 2015].

\section{Authors' information}

Avionics \& Control Laboratory,

Department of Aerospace sciences,

University of Beira Interior, 6201-001, Covilhã, Portugal.

E-mails: kawser.ah91@gmail.com bousson@ubi.pt

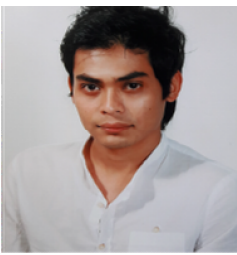

Kawser Ahmed received an Integrated Master's degrees in aeronautical engineering from University of Beira interior, Covilhã, Portugal in 2016. Currently is a PhD student in Department of Aerospace sciences at the University of Beira interior, Covilhã, Portugal, since September 2016. His current research includes optimal control, and trajectory optimization applied to fuel saving and environmental impact reduction. Eng. Ahmed is also a research member at the LAETA - Associated Laboratory for Energy, Transports, and Aeronautics.

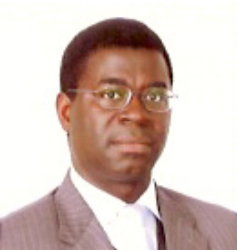

K. Bousson received a MEng degree in aeronautical engineering from the Ecole Nationale de l'Aviation Civile (ENAC) in 1988, a MSc degree in computer science (with emphasis on artificial intelligence) from Paul Sabatier University in 1989 , and a $\mathrm{PhD}$ degree in control \& computer engineering from the Institut National des Sciences Appliquées (INSA) in 1993, all in Toulouse, France. He was a researcher at the LAAS Laboratory of the French National Council for Scientific Research (CNRS) in Toulouse, from 1993 to 1995, and has been a professor in the Department of Aerospace Sciences at the University of Beira Interior, Covilhã, Portugal, since 1995, and member of LAETA (Associated Laboratory for Energy, Transports, and Aeronautics). His current research activities include trajectory optimization \& control of aerospace vehicles, nonlinear filtering and control of uncertain and chaotic systems, and machine learning. Dr. Bousson is a Member of the Portuguese Association for Automatic Control (APCA) and a Senior Member of the American Institute of Aeronautics and Astronautics (AIAA). 